\title{
Activity of ketoconazole against Mycobacterium tuberculosis in vitro and in the mouse model
}

\section{Correspondence \\ Ying Zhang \\ yzhang@jhsph.edu}

Received 9 November 2006

Accepted 10 April 2007

\author{
Sean T. Byrne, ${ }^{1} \dagger$ Steven M. Denkin, ${ }^{1} \dagger$ Peihua Gu, ${ }^{1}$ Eric Nuermberger ${ }^{2}$ \\ and Ying Zhang ${ }^{1}$
}

\author{
${ }^{1}$ Department of Molecular Microbiology and Immunology, Bloomberg School of Public Health, \\ Johns Hopkins University, Baltimore, MD 21205, USA \\ ${ }^{2}$ Center for Tuberculosis Research, School of Medicine, Johns Hopkins University, Baltimore, \\ MD 21231, USA
}

\begin{abstract}
There is an urgent need for the development of new drugs that are active against drug-resistant Mycobacterium tuberculosis strains and can shorten tuberculosis (TB) therapy. It has previously been reported that the azole class of antifungals has anti-TB activity in vitro. This study evaluated ketoconazole (KTC) for activity against M. tuberculosis. The MIC of KTC for different $M$. tuberculosis strains ranged from 8 to $16 \mu \mathrm{g} \mathrm{ml}^{-1}$ under both acidic and neutral conditions, with the minimum bactericidal concentration being about twofold higher than the MIC. KTC had enhanced activity against old, non-growing bacilli in vitro when combined with pyrazinamide (PZA) and rifampicin (RIF). A single oral dose of KTC at $75 \mathrm{mg} \mathrm{kg}^{-1}$ led to an inhibitory serum concentration $2 \mathrm{~h}$ after administration. The in vivo activity of KTC was evaluated in established pulmonary TB in the murine model, compared alone and in combination with isoniazid (INH), PZA and RIF. KTC alone exhibited little effect after short-term treatment, with a borderline bacteriostatic effect on spleen colony counts but not on lung counts. KTC, when added in combination with INH, PZA and RIF, significantly improved the treatment outcome in the lungs (compared with treatment with INH, PZA and RIF). The lowest numbers of bacilli in lungs were found in mice treated with KTC, PZA and RIF. Further investigation is necessary to determine the role of KTC in the treatment of TB.
\end{abstract}

\section{INTRODUCTION}

Mycobacterium tuberculosis, the causative agent of tuberculosis (TB), remains a serious global health concern despite the widespread use of chemotherapeutic drugs and BCG (bacille Calmette-Guérin) vaccination. The development of additional methods of TB control is imperative. Of particular importance is the shortening of the duration of therapy, as well as the introduction of new compounds effective against emerging drug-resistant strains of $M$. tuberculosis.

Successful treatment of TB involves targeting the multiple populations of bacteria that reside in the host. Currently, $\mathrm{TB}$ is treated with a combination of drugs including isoniazid (INH), rifampicin (RIF), pyrazinamide (PZA) and ethambutol given for 2 months, followed by a 4 month treatment with INH and RIF (American Thoracic Society et al., 2003). The lengthy therapy is often associated with

†These authors contributed equally to this work.

Abbreviations: INH, isoniazid; KTC, ketoconazole; PZA, pyrazinamide; $\mathrm{RIF}$, rifampicin; SIT, serum inhibitory titre; TB, tuberculosis. poor patient compliance and the emergence of drug resistance.

We have shown previously that members of the azole class of antifungal agents had significant anti-TB activity in vitro (Sun \& Zhang, 1999). In addition, an interesting property of the azole drugs is that they are active against growing bacilli and, to some extent, stationary-phase bacilli. This latter property may be important for shortening the duration of TB therapy by killing a population of bacilli not readily killed by current TB drugs. Of the azole drugs, ketoconazole (KTC) was selected for examination, as it is a commonly used, systemic drug (unlike some of the other azoles that are used topically). This study evaluated KTC for its activity against $M$. tuberculosis in vitro and in the mouse model.

\section{METHODS}

Bacterial strains. M. tuberculosis strains $\mathrm{H} 37 \mathrm{Rv}$ and $\mathrm{H} 37 \mathrm{Ra}$ were obtained from the ATCC and subcultured in Middlebrook 7H9 liquid medium (Becton Dickinson) with ADC (0.5\% BSA, $0.2 \%$ glucose, $0.0003 \%$ catalase, $0.08 \%$ sodium chloride), $0.2 \%$ glycerol and $0.05 \%$ Tween 80 (Sigma). In vivo studies made use of strain $\mathrm{H} 37 \mathrm{Rv}_{\mathrm{M}}$, a 
strain of H37Rv originally obtained from the ATCC, which was passaged twice in mice to ensure virulence and kept frozen in aliquots until infection.

MIC and minimum bactericidal concentration (MBC) determination. Twofold dilutions of each drug were prepared in 7H9 broth with ADC. Each tube was inoculated 1:10 with a $10^{-1}$ dilution of a 10- to 14-day-old culture (final concentration of about $10^{5}$ c.f.u.) of $\mathrm{H} 37 \mathrm{Rv}$ or PAS- and streptomycin-resistant H37Rv ATCC 35824. After 14 days, tubes were inspected for visible growth and c.f.u. counts were determined for those dilutions with no visible growth by plating on 7H11 agar with ADC. The MIC value was the drug concentration that completely inhibited growth. The $\mathrm{MBC}$ value was the minimum concentration that killed $99 \%$ of inoculated bacteria. Determination of MIC and MBC was performed under both neutral $(\mathrm{pH}=6.7-6.8)$ and acidic $(\mathrm{pH}=5.5-5.8)$ conditions.

Drug exposure assay. Two-month-old cultures of strain H37Ra were washed and resuspended in an equal volume of 7H9 broth without ADC or glycerol supplementation and exposed for 3 days to KTC $\left(1,5,10\right.$ or $\left.50 \mu \mathrm{g} \mathrm{ml}^{-1}\right)$, RIF $\left(10 \mu \mathrm{g} \mathrm{m}^{-1}\right)$ or PZA $(100 \mu \mathrm{g}$ $\mathrm{ml}^{-1}$ ), or combinations of RIF, PZA and KTC (Table 1). Drugs were dissolved in DMSO and diluted to a final concentration of $0.1 \%$ DMSO. Prior to inoculation, the medium was adjusted to a $\mathrm{pH}$ of 5.5-5.8 with $1 \mathrm{M} \mathrm{HCl}$ (Fisher Scientific). Solvent alone was used as a control. Following exposure for the indicated time, cells were washed and resuspended to remove the drug(s) and plated on 7H11 agar with $\mathrm{ADC}$ and $0.5 \%$ glycerol supplementation to enumerate the remaining live cells.

Determination of serum inhibitory titre (SIT) of the azole drug KTC. Eight-week-old Swiss mice (NCI) were administered KTC $\left(75 \mathrm{mg} \mathrm{kg}^{-1}\right)$, INH $\left(25 \mathrm{mg} \mathrm{kg}^{-1}\right)$ as a control or no drug in $0.2 \mathrm{ml}$ sterile water by oral gavage. Mice were then anaesthetized and exsanguinated $1-2 \mathrm{~h}$ after dosing. As the SIT for KTC in relation to M. tuberculosis had not been determined previously, the serum halflife was used as a guide for determining when to collect serum. Serum

Table 1. c.f.u. counts of 2-month-old cultures of $M$. tuberculosis H37Ra exposed for 3 days in vitro to KTC, PZA and RIF, alone and in combination

\begin{tabular}{|c|c|}
\hline Exposure condition & $\begin{array}{c}\text { Mean } \\
\log _{10}\left(\text { c.f.u. } \mathrm{ml}^{-1}\right) \pm S D^{*}\end{array}$ \\
\hline Control & $5.88 \pm 0.03$ \\
\hline PZA $\left(100 \mu \mathrm{g} \mathrm{ml}^{-1}\right)$ & $5.67 \pm 0.06 \dagger$ \\
\hline $\operatorname{RIF}\left(10 \mu \mathrm{g} \mathrm{ml}^{-1}\right)$ & $4.60 \pm 0.24 \dagger$ \\
\hline $\mathrm{PZA}\left(100 \mu \mathrm{g} \mathrm{ml}^{-1}\right)+\mathrm{RIF}\left(10 \mu \mathrm{g} \mathrm{ml}^{-1}\right)$ & $4.67 \pm 0.19 \dagger$ \\
\hline $\mathrm{KTC}\left(1 \mu \mathrm{g} \mathrm{ml}^{-1}\right)$ & $5.78 \pm 0.12$ \\
\hline $\mathrm{KTC}\left(1 \mu \mathrm{g} \mathrm{ml}^{-1}\right)+\mathrm{PZA}+\mathrm{RIF}$ & $4.14 \pm 0.05 \ddagger$ \\
\hline $\mathrm{KTC}\left(5 \mu \mathrm{g} \mathrm{ml}^{-1}\right)$ & $5.67 \pm 0.16$ \\
\hline $\mathrm{KTC}\left(5 \mu \mathrm{g} \mathrm{ml}^{-1}\right)+\mathrm{PZA}+\mathrm{RIF}$ & $4.37 \pm 0.28$ \\
\hline $\mathrm{KTC}\left(10 \mu \mathrm{g} \mathrm{ml}^{-1}\right)$ & $5.70 \pm 0.17$ \\
\hline $\mathrm{KTC}\left(10 \mu \mathrm{g} \mathrm{ml}^{-1}\right)+\mathrm{PZA}+\mathrm{RIF}$ & $3.80 \pm 0.08 \S$ \\
\hline $\mathrm{KTC}\left(50 \mu \mathrm{g} \mathrm{ml}^{-1}\right)$ & $4.00 \pm 0.30 \ddagger$ \\
\hline $\mathrm{KTC}\left(50 \mu \mathrm{g} \mathrm{ml}^{-1}\right)+\mathrm{PZA}+\mathrm{RIF}$ & $<2.00 \$$ \\
\hline
\end{tabular}

${ }^{\star}$ In this assay, the lower limit of detection was $2.00 \log _{10}$ (c.f.u. $\mathrm{ml}^{-1}$ ). $\dagger P<0.005$ compared with the control group.

$\ddagger P<0.05$ compared with the PZA + RIF group.

$\S P<0.005$ compared with the PZA + RIF group. was prepared and samples from each group were pooled. An equal volume of serum $(2.5 \mathrm{ml})$ was added to $2.5 \mathrm{ml} 7 \mathrm{H} 9$ broth with ADC supplement and twofold dilutions were prepared, into which $0.01 \mathrm{ml}$ exponential-phase $\mathrm{H} 37 \mathrm{Rv}$ was inoculated. These cultures were then incubated at $37{ }^{\circ} \mathrm{C}$ and inspected for growth 14 days later to assess the serum concentration of drugs that inhibited bacterial growth. Animal protocols were approved by the John Hopkins University Animal Care and Use Committee.

Mouse infections. Infections were conducted using a method adapted from Nuermberger et al. (2004). Four- to six-week-old female $\mathrm{BALB} / \mathrm{c}$ mice (NCI) were infected with high-dose, aerosolized $M$. tuberculosis. A frozen stock of mouse-passaged H37Rv was cultured in 7H9 broth with $\mathrm{ADC}$ and glycerol and incubated for 14 days at $37^{\circ} \mathrm{C}$ before use in a Middlebrook Inhalation Exposure System (Glas-Col).

Treatments. Following infection, mice were randomized to a treatment group (four to five mice per group). To allow for established infections, therapy was initiated 16 days after aerosol infection and administered 5 days per week for a month. Drugs were suspended in $0.2 \%$ agarose and administered by oral gavage in a total volume of $0.2 \mathrm{ml}$. Drugs in combination were administered simultaneously; one control group received carrier only.

Drugs were used in the experiments at the following concentrations: KTC, $75 \mathrm{mg} \mathrm{kg}^{-1}$; INH, $25 \mathrm{mg} \mathrm{kg}^{-1}$; RIF, $10 \mathrm{mg} \mathrm{kg}^{-1}$; and PZA, $150 \mathrm{mg} \mathrm{kg}^{-1}$ (compounds obtained from Sigma). Stock solutions of these drugs were stored at $-20{ }^{\circ} \mathrm{C}$ between treatment sessions.

Determination of c.f.u. The day after the conclusion of treatment, mice were euthanized. Total lungs and spleens were homogenized in $2 \mathrm{ml}$ PBS. Serial dilutions of $10^{0}, 10^{-2}, 10^{-4}$ and $10^{-6}(250 \mu \mathrm{l})$ were plated on Middlebrook 7H11 agar (Becton Dickinson) supplemented with ADC (Becton Dickinson) and PANTA (Becton Dickinson) to enumerate the total c.f.u. of $M$. tuberculosis per organ per mouse. c.f.u. counts from one group of controls were determined at the start of treatment to establish a baseline of infection; c.f.u. counts were also obtained from control mice at the conclusion of the study.

Statistical analyses. c.f.u. counts were converted to $\log _{10}$ values and compared using Student's $t$-test.

\section{RESULTS AND DISCUSSION}

\section{MIC and MBC values of KTC against M. tuberculosis strains}

The azole class of antifungals including miconazole and clotrimazole was previously identified by our laboratory as having in vitro anti-TB activity (Sun \& Zhang, 1999). As miconazole and clotrimazole are topical agents, we evaluated the activity of KTC against $M$. tuberculosis in this study. Itraconazole and fluconazole had no significant anti-TB activity in vitro (unpublished data). The MIC of KTC against virulent $M$. tuberculosis $\mathrm{H} 37 \mathrm{Rv}$ in liquid medium was $8-16 \mu \mathrm{g} \mathrm{ml}^{-1}$ and the MBC was $32 \mu \mathrm{g} \mathrm{ml}^{-1}$. The MIC of KTC against strain H37Ra in antibioticcontaining solid medium was found to be $8 \mu \mathrm{g} \mathrm{ml}^{-1}$ and the MBC was $16 \mu \mathrm{g} \mathrm{ml}^{-1}$. The MIC and MBC determinations for H37Rv and H37Ra were performed at both neutral and acidic $\mathrm{pH}$ values and similar values were found under both conditions. 


\section{Synergy of KTC with PZA and RIF in vitro}

In TB infection, it is thought that a subpopulation of bacteria, originally susceptible to antibiotics, is able to persist in tissues despite exposure to drugs (McCune et al., 1957). A compound that targets this subset of cells may be able to shorten the duration of therapy. The adoption of PZA, which has been shown to be active against non-replicating persisting bacilli in vitro (Zhang et al., 2002), into the regimen for $\mathrm{TB}$ treatment has partly been responsible for a shortening in the duration of therapy. Through a drug exposure assay that measured activity against non-growing bacteria, KTC was found to exhibit a synergistic effect with RIF and PZA after a 3 day exposure in $7 \mathrm{H} 9$ broth without ADC, pH 5.5 (Table 1). Two-month-old $M$. tuberculosis H37Ra cultures were exposed for 3 days to KTC $\left(1,5,10\right.$ or $\left.50 \mu \mathrm{g} \mathrm{ml}^{-1}\right)$, RIF $\left(10 \mu \mathrm{g} \mathrm{ml}^{-1}\right)$ or PZA $\left(100 \mu \mathrm{g} \mathrm{ml}^{-1}\right)$, or combinations of KTC with RIF and PZA under acidic conditions ( $\mathrm{pH}$ 5.5). RIF was used as a positive control instead of INH because the culture used for drug exposure was 2 months old and it is known that old cultures are not susceptible to INH but are more susceptible to RIF. No synergy was detected following short-term exposure against exponential-phase M. tuberculosis (data not shown).

KTC at a concentration of $50 \mu \mathrm{g} \mathrm{ml}^{-1}$ exhibited significant anti-TB activity during a short-term exposure on nongrowing cells, reducing viable bacterial counts from $5.88 \log _{10}$ (c.f.u. $\mathrm{ml}^{-1}$ ) (control) to $4.00 \log _{10}$ (c.f.u. $\mathrm{ml}^{-1}$ ) (Table 1). This decrease was greater than that seen with RIF or PZA alone or in combination. Furthermore, considerable anti-TB activity was observed when KTC $\left(50 \mu \mathrm{g} \mathrm{ml}^{-1}\right)$ was combined with RIF and PZA. After exposure to these three drugs for 3 days, no viable bacteria could be cultured [lower limit of detection was $2 \log _{10}$ (c.f.u. $\mathrm{ml}^{-1}$ )].

Lower concentrations of $\operatorname{KTC}\left(1,5\right.$ and $\left.10 \mu \mathrm{g} \mathrm{ml}^{-1}\right)$ were found to have minimal effects on the non-growing population of TB after a 3 day exposure (reduction in c.f.u. values of less than tenfold). However, when combined with RIF and PZA, these lower concentrations of KTC showed greater anti-TB activity than RIF or PZA alone (Table 1).

The mode of action of KTC may be due to inhibition of $M$. tuberculosis cytochrome P450 mono-oxygenases (McLean et al., 2002). Azole antifungals have been shown to bind to the P450s CYP121 and CYP51 in M. tuberculosis and may exert their antimycobacterial properties by targeting these molecules (McLean et al., 2002). Azoles have also been found to inhibit the tricarboxylic acid cycle enzyme succinate dehydrogenase (Boshoff et al., 2004). The enhancement of PZA activity by KTC seen in this study could be related to the inhibition of succinate dehydrogenase by KTC such that it makes it easier for PZA, which depletes membrane energy, to kill tubercle bacilli. Further investigation into the mechanism of action of KTC on $M$. tuberculosis is needed.

\section{Serum inhibitory testing}

Before drug assessment in vivo, serum inhibitory testing was performed. Serum inhibitory testing is an in vitro assay that combines pharmacodynamic and pharmacokinetic principles to determine the antibacterial activity of a drug in the serum following administration (NCCLS, 1999). A single $75 \mathrm{mg} \mathrm{kg}^{-1}$ dose of KTC resulted in a serum concentration that was able to inhibit the growth of $M$. tuberculosis $2 \mathrm{~h}$ after oral administration. The SIT is the lowest dilution of serum that inhibits growth of M. tuberculosis in vitro. At the given dose, KTC was found to have an SIT of $1: 2$ to $1: 4$ against $M$. tuberculosis.

\section{Dose optimization in unestablished murine infection}

To optimize oral dosing of KTC in an in vivo murine model of unestablished infection, 4-week-old, female BALB/c mice were infected with an aerosolized, exponential-phase culture of a mouse-adapted strain of M. tuberculosis H37Rv. Treatment was initiated the day after infection and continued for 5 days per week for 4 weeks. By employing a dosing study, KTC at $75 \mathrm{mg} \mathrm{kg}^{-1}$ was found to be the lowest daily dose with activity against unestablished, murine $\mathrm{TB}$ infection, in a manner similar to treatment with INH alone (data not shown). Daily administration with this dose for 1 month was found to have no overt adverse effects in the infected animals.

\section{Activity of KTC after short-duration treatment of established TB in the mouse model}

The efficacy of KTC in combination with currently used TB drugs was assessed in a murine model of established pulmonary TB. KTC was co-administered daily for 1 month with various combinations of INH, PZA and RIF. Following treatment, the total number of c.f.u. in lung and spleen tissues was determined (Table 2).

On the day of treatment initiation (day 16 post-infection), signs of a well-established infection were present in the lungs and spleen of mice, and by day 44 post-infection, at treatment discontinuation, the number of bacteria increased by about $0.8 \log _{10}$ c.f.u. in the lungs and $1.8 \log _{10}$ c.f.u. in the spleen. After 1 month of treatment with the positive-control RIF only, there was a decrease in the number of bacilli in the lungs and spleen compared with untreated mice. Treatment with KTC only for 1 month led to a slight bacteriostatic effect in the spleen $(P<0.01$, compared with untreated mice at the conclusion of the study), but no significant change in lung counts was observed $(P>0.05)$ (Table 2).

The group treated with the combination of INH, PZA and RIF had lung counts that were about $3 \log _{10}$ c.f.u. less than those of the controls at the beginning of treatment and almost $4 \log _{10}$ c.f.u. less than those of the controls at the end of the study $(P<0.001)$. Mice receiving INH, PZA and 
Table 2. Lung and spleen c.f.u. counts at 44 days after treatment, which was initiated at 16 days post-inoculation

\begin{tabular}{|llc|}
\hline \multirow{2}{*}{ Treatment } & \multicolumn{2}{c|}{ Mean $\log _{\mathbf{1 0}}$ c.f.u. \pm SD $^{*}$} \\
\cline { 2 - 3 } & \multicolumn{1}{c|}{ Lung } & Spleen \\
\hline Untreated (initial count) $\dagger$ & $6.71 \pm 0.16$ & $3.22 \pm 0.13$ \\
Untreated (end count) $\dagger$ & $7.52 \pm 0.54$ & $5.01 \pm 0.09$ \\
RIF & $5.66 \pm 0.26 \ddagger$ & $3.23 \pm 0.65 \ddagger$ \\
KTC & $7.37 \pm 0.13$ & $4.69 \pm 0.16 \ddagger$ \\
INH + PZA + RIF & $3.63 \pm 0.18 \ddagger$ & $<0.90 \ddagger$ \\
KTC + INH + PZA + RIF & $3.29 \pm 0.13 \$$ & $<0.90$ \\
KTC + INH + PZA & $4.91 \pm 0.33 \|$ & $1.08 \pm 0.84$ \\
KTC + INH + RIF & $3.57 \pm 0.09$ & $<0.90$ \\
KTC + PZA + RIF & $2.78 \pm 0.54 \|$ & $<0.90$ \\
& & \\
\hline
\end{tabular}

${ }^{\star}$ In this experiment, the lower limit of detection was $0.90 \log _{10}$ c.f.u. $\dagger$ c.f.u. counts were determined for untreated controls at day 16 (initial count) and at day 44 (end count).

$\ddagger P<0.01$ compared with the untreated group at study conclusion. $\$ P<0.05$ compared with the INH, PZA, RIF group. $\| P<0.01$ compared with the INH, PZA, RIF group.

SPositive spleen count in one animal; all other spleens in this group were negative.

RIF exhibited complete clearance of detectable bacilli from the spleen (Table 2).

The addition of KTC to INH, PZA and RIF resulted in a further decrease in the number of bacteria in the lungs compared with the INH, PZA and RIF group $(P<0.05)$ and negative spleen counts.

Treatment with KTC, INH and RIF (replacement of PZA with KTC) led to a slight but insignificant decrease in the lung c.f.u. counts compared with the INH, PZA and RIF group $(P>0.05)$. The effect of KTC, INH and RIF was essentially indistinguishable from the INH, PZA and RIF treatment group after 1 month of treatment in this model.

Mice given KTC, INH and PZA (replacement of RIF with KTC) had higher lung counts than the INH, PZA and RIF group, and one mouse in the group had a spleen positive for M. tuberculosis, whilst the rest were negative (Table 2).

The lowest numbers of bacilli in lungs were found in the group administered KTC, PZA and RIF. When KTC replaced INH in a 1 month combination therapy, there was a decrease of about $4 \log _{10}$ c.f.u. per lung compared with the starting untreated controls and about $4.7 \log _{10}$ c.f.u. per lung compared with the end count of untreated controls. The lung c.f.u. value for the KTC, PZA and RIF group was significantly less than that for the INH, PZA and RIF group $(P<0.01)$ and also less than that for the KTC, INH, PZA and RIF group $(P<0.05)$ (Table 2$)$.

Treatment of established TB infection in animal models, as well as in patients, requires long-duration therapy. This study showed that the addition of KTC to the standard regimen of INH, PZA and RIF resulted in increased killing of bacilli in the first month of treatment. However, the observed enhancement of bacterial clearance after 1 month of therapy, whilst statistically significant, was not dramatic; it remains to be seen whether the addition of KTC would translate into a shorter time to cure in a longer treatment study. Interestingly, replacement of INH in a three-drug regimen with KTC led to an even greater reduction in the number of viable bacilli in infected animals. This phenomenon may be related to the well-known antagonism between INH and PZA (Grosset et al., 1992). As coadministration of KTC with RIF has been reported to lower the serum concentrations of RIF (Finch et al., 2002) and KTC (Engelhard et al., 1984) compared with administration of each drug separately, future studies may wish to examine the administration of higher doses of RIF or KTC to compensate for the pharmacokinetic interaction, even though a normal dose of RIF was shown to have an effect when combined with KTC in this study.

This account is the first report of the potential anti-TB activity of KTC in vivo. An enhancement of anti-TB activity was observed in vitro against non-growing $M$. tuberculosis when KTC was combined with PZA and RIF. The results from short-course treatment in this mouse model indicate that replacement of INH in the standard regimen with KTC may lead to increased killing of bacteria. These results are preliminary in nature and further investigations are underway to examine the effect of KTC in long-term treatment of $M$. tuberculosis infection in the mouse model.

\section{ACKNOWLEDGEMENTS}

The authors wish to thank Katrin Post-Martens, Sandeep Tyagi, Kathy Williams and Jacques Grosset for assistance with the mouse model. This study was supported by NIH grants AI44063 and AI49485.

\section{REFERENCES}

American Thoracic Society, CDC \& Infectious Diseases Society of America (2003). Treatment of tuberculosis. MMWR Recomm Rep 52 (RR11), 1-77.

Boshoff, H. I., Myers, T. G., Copp, B. R., McNeil, M. R., Wilson, M. A. \& Barry, C. E., III (2004). The transcriptional responses of Mycobacterium tuberculosis to inhibitors of metabolism: novel insights into drug mechanisms of action. J Biol Chem 279, 40174-40184.

Engelhard, D., Stutman, H. R. \& Marks, M. I. (1984). Interaction of ketoconazole with rifampin and isoniazid. N Engl J Med 311, 1681-1683.

Finch, C. K., Chrisman, C. R., Baciewicz, A. M. \& Self, T. H. (2002). Rifampin and rifabutin drug interactions: an update. Arch Intern Med 162, 985-992.

Grosset, J., Truffot-Pernot, C., Lacroix, C. \& Ji, B. (1992). Antagonism between isoniazid and the combination pyrazinamide-rifampin against tuberculosis infection in mice. Antimicrob Agents Chemother 36, 548-551.

McCune, R., Lee, S. H., Deuschle, K. \& McDermott, W. (1957). Ineffectiveness of izoniazid in modifying the phenomenon of microbial persistence. Am Rev Tuberc 76, 1106-1109.

McLean, K. J., Marshall, K. R., Richmond, A., Hunter, I. S., Fowler, K., Kieser, T., Gurcha, S. S., Besra, G. S. \& Munro, A. W. (2002). Azole 
antifungals are potent inhibitors of cytochrome $\mathrm{P} 450$ monooxygenases and bacterial growth in mycobacteria and streptomycetes. Microbiology 148, 2937-2949.

NCCLS (1999). Methodology for the Serum Bactericidal Test; Approved Guideline. NCCLS document M21-A. Wayne, PA: National Committee for Clinical Laboratory Standards.

Nuermberger, E. L., Yoshimatsu, T., Tyagi, S., O'Brien, R. J., Vernon, A. N., Chaisson, R. E., Bishai, W. R. \& Grosset, J. H. (2004).
Moxifloxacin-containing regimen greatly reduces time to culture conversion in murine tuberculosis. Am J Respir Crit Care Med 169, 421-426.

Sun, Z. \& Zhang, Y. (1999). Antituberculosis activity of certain antifungal and antihelmintic drugs. Tuber Lung Dis 79, 319-320.

Zhang, Y., Permar, S. \& Sun, Z. (2002). Conditions that may affect the results of Mycobacterium tuberculosis susceptibility testing to pyrazinamide. J Med Microbiol 51, 42-49. 